\title{
Research the Orem Self-care Theory on the Aged Patients with Acute
}

\section{Myocardial Infarction}

\author{
Xueyan Chen ${ }^{1}$,Ling Gong ${ }^{1 *}$, Liping Pan $^{2}$ \\ 1. Nursing College,Beihua University.Jilin City. Jilin Province.China \\ 2.Cardiovascular Department of Beihua University Affiliated Hospital.Jilin City. Jilin Province.China \\ chenxueyan1976@sohu.com \\ Ling Gong is corresponding author
}

Keywords:Orem self-care theory, the aged patients, acute myocardial infarction

Abstract.Purpose In order to help the aged acute myocardial infarction (AMI) patients to take an active part in disease assessment, monitoring, treatment and improve the compliance, the AMI patients can relieve their somatic symptoms effectively, and at meanwhile, the incidence of complications can also be reduced.Method Two groups of patients were treated by routine treatment. Basing on the holistic nursing, the experimental group were adopted self-care theory to estimate the self-care ability of patients and their relatives. Patients and their relatives jointly participate in nursing activity by using different systems of care and health education. The control group were adopted the sequent nursing procedure to implement the holistic nursing.Results After leaving the hospital, the self-care ability of the experimental group was significantly higher than that of control group. The experimental group relieved their somatic symptoms effectively, and also reduce complications.Conclusion Orem self-care theory was applied in the treatment of aged acute myocardial infarction (AMI) patients, the patients take active part in treatment and nursing instead of passive treatment, the patients' own value can be reflect fully, and better results were also achieved.

\section{Introduction}

Acute myocardial infarction (AMI) is the serious type of coronary heart disease, it has high incidence of crime and mortality. Recenly, as Medical level standards up and mortality sharp down, so does the stronger demand for AMI nursing. It has become the problems for medical staff to be solved,that is how to improve the patients' ability of living independence and quality of life. Orem,the famous American Nursing experts put forwards self-care theory, that is, through the assessment of the patient's self-care needs, self-care ability and basic requirements, and also comparison of self-care needs and self-care ability, we can determine self-care deficit, design the relevant nursing system, make specific plan, encourage patients and their relatives take part in nursing activities together, thus, patients' self-care ability can be enhances, and they can further restore, maintain and promote their health ${ }^{[1]}$. We has applied this guiding ideology and theory into nursing the aged patients with acute myocardial infarction care, and achieved significant effect. Sum up as follows now, 


\section{Data and Methods}

\section{Common data}

We selected 100 aged patients with acute myocardial infarction which were in cardiovascular department of Beihua University Affiliated Hospital during Jan.2010-Dec.2011, among them, male 74 cases, female 26 cases, they are aged 60-87 years old. After they were in hospital, on the basis of clinical symptoms, neurological examination and ECG typical, they were all diagnosed with myocardial infarction according to the year of 1979 WHO "On the Diagnosis of Ischemic Heart Disease and Naming Standard", of this, 38 cases of Anterior myocardial infarction, 29 cases of Inferior wall myocardial infarction, 16 cases of posterior wall myocardial infarction and 17 cases of extensive myocardial infarction. In the chronological order, we divided them into experimental group and control group, 50 cases in experimental group of 35 males and 15 females with average years of $66.98 \pm 9.03,50$ cases in control group of 37 males and 13 females with average years of 67.08 \pm 9 . 15. Though there were no statistical significance between the two groups at sex, age, cultural degree and extent of lesions $(\mathrm{P}>0.05)$, it has comparability with the average hospitalization time being 21 days. The Discharged patients of acute myocardial infarction were all made an investigation of the follow-up survey on one year.

\section{Methods}

Two groups of patients were treated by routine treatment. Basing on the holistic nursing, the experimental group were adopted self-care theory to estimate the self-care ability of patients and their relatives. Patients and their relatives jointly participate in nursing activity by using different systems of care and health education. The control group were adopted the sequent nursing procedure to implement the holistic nursing.

\section{Assessment and nursing}

The assessment of project includes changes of common self-care needs, changes of development self-care needs, and also changes of self-care needs at poor health. We can provide the corresponding nursing system according to the different demand degree of aged patients and their relatives.

\section{Complete compensation system}

Being with the rapid development, the aged patients with acute critical condition will loss their self-care abilities completely, thus, the nurses should provide their patients comprehensive assistance, in order to meet the patients' self-care demands.Complete compensation system includes oxygen, intravenous administration, nutrition, excretion, personal hygiene etc.The specific steps were as the follows: (1)keeping at modified bedrest, (2) giving oxygen inhalation, keeping the patients at easy breathing, (3)establishing intravenous access for patients, providing various drugs at doctor's advices and giving close observation at he patient's consciousness, (4) giving closely observe at patients' consciousness, vital signs and urine volume changes, and also giving a 24- hour accurate record, 5providing the reasonable diet and helping the patients with their daily life and activities, such as oral care 2-3 times / day; 6keeping patients' bowels open, if necessary, giving a laxative or cleaning enema so that the patients defecation breathless can be avoided, (7)strengthening psychological nursing, encouraginge the patients to express their inner feelings, at meanwhile, we should give the timely comfort so that the patients nervousness, apprehensiveness were eliminated and good cooperation to the treatment was obtained.

\section{Partial compensation system}

Being at the AMI subacute period, patients with partial capacity to meet their therapeutic self-care 
demands, nurses and patients also with their relatives should share patient self-care activities, the nurses should act as educators and caretakers instead of the former caretakers. The nurses should manage the nursing plan with patients and their relatives, on the other hand, carrying out the nursing measures, assist patients increasing activity contents gradually and earning how to self care patients by themselves.

For the aged AMI patients, the nurses should be kind and considerate, patients show more empathy, compassion and respect towards the needs. The aged patients show behavioral disorders on a sudden illness and totally incapacitated by the illness, thus, they need warm and thoughtful, meticulous nursing care ${ }^{[2]}$. The nursers should strengthen the communication with the patients, so as to establish its treatment of optimal mental state.

\section{Auxiliary educational system}

At any period of illness, nursers should frequently provide patients and their relatives relevant information and emotional support ${ }^{[3]}$. We evaluate patients psychological needs, self-care ability, family support system at different stages, according to the demand for psychological care, we put forward psychological nursing to meet the patients demands, thus, patients and their relatives can understand the current condition. During the acute phase, we introduce the ward environment, doctors and nurses and the purpose of treatment. We tell the patients to relax the tense mood, and also tell them that the bad mood may induce arrhythmias, acute myocardial ischemia. In subacute period, we Introduce the relevant knowledge of the disease, such as common etiology, pathogenesis, knowledge of rehabilitation and give specific guidance. During the rehabilitative stage, we tell the patients should be treated as Coronary heart disease, take medicines at a physician's, have the regular reexamination. We should teach patients health knowledge of self care, such as alcohol, emotional stability, maintain defecate unobstructed etc,so, we can prevent the occurrence of angina or recurrence of AMI. At the same time, the patients relatives should play an important role in helping and caring the patients.

Statistical method By using SPSS12.0 for statistical analysis, we find the difference of $\mathrm{P}<0.05$ with statistical significance.

\section{Results}

\section{Self-care ability comparisons between two groups after six-month discharge （Table 1）}


Complication comparisons between two groups after six-month discharge （Table 2）

Table2 Complication comparisons between two groups after six-month discharge(No.)

\begin{tabular}{|c|c|c|c|c|c|c|c|c|}
\hline group & & $\begin{array}{r}\text { Angi } \\
\text { pectoris }\end{array}$ & & arrhythmia & $\begin{array}{l}\text { Heart } \\
\text { failure }\end{array}$ & AMI & $\begin{array}{l}\text { Recurrent } \\
\text { sudden death }\end{array}$ & cardiac \\
\hline $\begin{array}{l}\text { experimental } \\
\text { group }\end{array}$ & $(50)$ & 1 & 3 & & 2 & & 2 & 1 \\
\hline Control group & $(50)$ & 3 & 11 & & 9 & & 8 & 6 \\
\hline$x^{2}$ & & 1.04 & 5.32 & & .01 & & 4.00 & 3.84 \\
\hline $\mathrm{P}$ & & $>0.05$ & $<0.05$ & & $<0.05$ & & $<0.05$ & $<0.05$ \\
\hline
\end{tabular}

\section{Conclusion}

Orem self-care theory emphasizes the importance of self-care ability, believes that self-care is a kind of purposeful activity learned from others, while during the completion of care self, we need wisdom, experience, guidance and help from others. Which can be seen in Table 1, the experimental group ability was significantly higher than the control group after discharge from hospital, at the different development stages of AMI, we provide patients and their relatives different intervention from Physiological, behavioral,and psychological conditions. It is effective for the patients to enhance their self-care ability, esp. at Partial compensation system and auxiliary educational system.It mobilize the energies of patients and their families, realize the self care to a great extent, and also improve the quality of life. At meanwhile, due to the complex development process of AMI, we adopt the different nursing modes on the different periods of AMI patients. From table 2, we can see that Orem self-care theory can reduce the occurrence of complications, as well as relieve somatic symptoms effectively.

Orem self-care theory combines science, art and technology, its core lies in that it can encourage patients take part in disease assessment, monitoring, treatment and improve the compliance. Especially for the aged patients, through self care, they take part in their medical activities and decisions at th equal status, not only meet the needs of their self-esteem and self-confidence, at the same time, through self-directed and self-care behavior, they form the healthy lifestyle and promote their health ${ }^{[4]}$.

Orem self-care theory also enable the nursers become the health promotion and publicity in stead of the former simple and repetitive technique operators. Nursers have the opportunity to express their professional knowledge. Through the guidance and education to the patients, and also training the restoration of normal self-care ability, the responsibility of nursers have been raised to a higher level, it encourage nursers to research experiment and explore their nursing works ${ }^{[5]}$, it is also in favor of the enhancement of nurses' professional level and to the continuous development of nursing specialty.

\section{References}

[1] Xianqiong Feng. Nursing Introduction.Beijing : People's Medical Publishing House, 2006: 65 [2]Chuanhua Shen. Application of Orem self-care model on the aged patients.Nursing Research,2008,2(6B):1618-1619 
[3] Cuiqi Wen. Using self-care theory to guide patients of hilar bile duct carcinoma comprehensive treatment. Journal of Chinese Nursing,2000,35 (2): 92-94

[4] Jianpeng Wang, Yuchun Liu, Yanlan Ma.Geriatric nursing new ideas and the elderly special issues in nursing. Journal of Chinese Nursing,,2003, 38 (12): 959

[5] Qiuyun Mao, Aiqin Sun, Bo Ning, etc. The application of Orem self-care theory on Cerebral hemorrhage patients postoperative recovery period. Journal of Chinese Nursing,2004, 39 (12): 96-97. 